\title{
Identification and expression analysis of cytokinin metabolic genes IPTs, CYP735A and CKXs in the biofuel plant Jatropha curcas
}

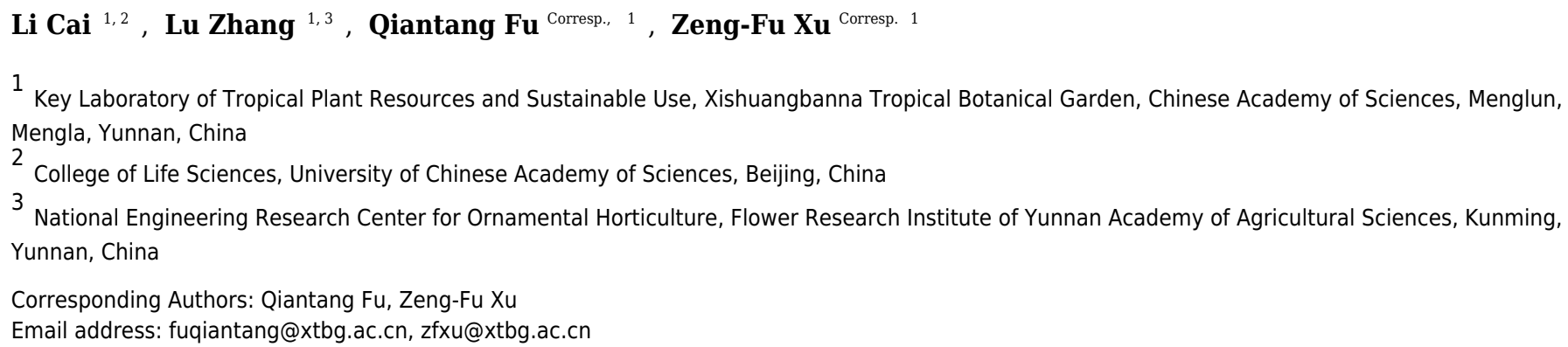

The seed oil of Jatropha curcas is considered a potential bioenergy source that could replace fossil fuels. However, the seed yield of Jatropha is low and has yet to be improved. We previously reported that e xogenous cytokinin treatment increased the seed yield of Jatropha . Cytokinin levels are directly regulated by isopentenyl transferases (IPT), cytochrome P450 monooxygenase (CYP735A) and cytokinin oxidase/dehydrogenase (CKX). In this study, we cloned six IPT genes, one JCCYP735A gene, and seven JCCKX genes. The expression patterns of these 14 genes in various organs were determined using real-time quantitative PCR. JCIPT1 was primarily expressed in roots and seeds , JCIPT2 was expressed in roots, apical meristems, and mature leaves, JCIPT3 was expressed in stems and mature leaves, JCIPT5 was expressed in roots and mature leaves, JCIPT6 was expressed in seeds at 10 days after pollination, and JCIPT9 was expressed in mature leaves. JCCYP735A was mainly expressed in roots, flower buds, and seeds. The seven JCCKX genes also showed different expression patterns in different organs of Jatropha. In addition, CK levels were detected in flower buds and seeds at different stages of development. The concentration of iP, iPR and tZ increased with flower development, and the concentration of iP decreased with seed development, while that of $t Z$ increased. We further analyzed the function of JCCYP735A using the CRISPR-Cas9 system, and found that the concentrations of $t Z$ and tZR decreased significantly in the Jccyp735a mutants, which showed severely retarded growth. These findings will be helpful for further studies of the functions of cytokinin metabolic genes and understanding the roles of cytokinins in Jatropha growth and development. 
1 Identification and expression analysis of cytokinin metabolic 2 genes IPTs, CYP735A and CKXs in the biofuel plant Jatropha 3 curcas

4

5 Li Cai ${ }^{1,2}$, Lu Zhang ${ }^{1,3}$, Qiantang $\mathrm{Fu}^{1,{ }^{1,}}$, Zeng-Fu Xu ${ }^{1, *}$

6

7 1. Key Laboratory of Tropical Plant Resources and Sustainable Use, Xishuangbanna Tropical

8 Botanical Garden, Chinese Academy of Sciences, Menglun, Yunnan 666303, China;

9 2. College of Life Sciences, University of Chinese Academy of Sciences, Beijing, China

10 3. National Engineering Research Center for Ornamental Horticulture, Flower Research

11 Institute of Yunnan Academy of Agricultural Sciences, Kunming, Yunnan 650205, China

12

13 * Corresponding Authors:

14 Qiantang $\mathrm{Fu}^{1}$, fuqiantang@xtbg.ac.cn;

$15 \quad$ Zeng-Fu Xu ${ }^{1}, \underline{\text { zfxu@Xtbg.ac.cn }}$ 


\section{ABSTRACT}

The seed oil of Jatropha curcas is considered a potential bioenergy source that could replace fossil fuels. However, the seed yield of Jatropha is low and has yet to be improved. We previously reported that exogenous cytokinin treatment increased the seed yield of Jatropha. Cytokinin levels are directly regulated by isopentenyl transferases (IPT), cytochrome P450 monooxygenase (CYP735A) and cytokinin oxidase/dehydrogenase (CKX). In this study, we cloned six IPT genes, one $J_{c} C Y P 735 A$ gene, and seven $J_{c} C K X$ genes. The expression patterns of these 14 genes in various organs were determined using real-time quantitative PCR. JCIPT1 was primarily expressed in roots and seeds, JcIPT2 was expressed in roots, apical meristems, and mature leaves, JcIPT3 was expressed in stems and mature leaves, JcIPT5 was expressed in roots and mature leaves, JcIPT6 was expressed in seeds at 10 days after pollination, and JcIPT9 was expressed in mature leaves. $J c C Y P 735 A$ was mainly expressed in roots, flower buds, and seeds. The seven $J_{C} C K X$ genes also showed different expression patterns in different organs of Jatropha. In addition, CK levels were detected in flower buds and seeds at different stages of development. The concentration of $\mathrm{iP}, \mathrm{iPR}$ and $\mathrm{tZ}$ increased with flower development, and the concentration of iP decreased with seed development, while that of $\mathrm{tZ}$ increased. We further analyzed the function of $J_{C} C Y P 735 A$ using the CRISPR-Cas9 system, and found that the concentrations of $\mathrm{tZ}$ and tZR decreased significantly in the Jccyp 735 a mutants, which showed severely retarded growth. These findings will be helpful for further studies of the functions of cytokinin metabolic genes and understanding the roles of cytokinins in Jatropha growth and development. 
43

44

\section{INTRODUCTION}

Jatropha curcas is a multipurpose tree that belongs to the Euphorbiaceae family. It can endure drought and adapt to barren land in tropical and subtropical regions. Jatropha is considered a promising biofuel plant due to the high oil content in its seeds (Akashi 2012; Francis et al. 2005; Makkar \& Becker 2009). However, the seed yield is very low, potentially because of the relatively low number of total flowers and/or the ratio of female to male flowers in Jatropha (Kumar \& Sharma 2008; Kumar Tiwari et al. 2007; Rao et al. 2008). Recently, several studies have reported that exogenous cytokinin (CK) treatment can significantly increase the total number of flowers per inflorescence, the female-to-male flower ratio, and the seed yield (Fröschle et al. 2017; Pan \& Xu 2011).

CKs are important hormones in plants and participate in many biological processes, such as apical dominance (Shimizu-Sato et al. 2009; Tanaka et al. 2006), root proliferation (Kudo et al. 2010; Werner et al. 2003), reproductive development (Ashikari et al. 2005), and senescence (Gan \& Amasino 1995). Endogenous CKs containing N6-( $\Delta^{2}$ - isopentenyl)-adenine (iP), trans-zeatin (tZ), cis-zeatin (cZ), dihydrozeatin (DZ), and their conjugates are known as isoprenoid CKs (Mok \& Mok 2001). The major derivatives are generally iP- and tZ-type CKs (Sakakibara 2006).

CK biosynthesis and degradation pathways have been well studied in the past decade (Figure 1) (Galuszka et al. 2007; Kudo et al. 2010; Sakakibara 2006). The first step of iP and tZ biosynthesis is catalysed by adenosine phosphate-isopentenyltransferases (IPTs). IPTs produce iP-ribotides from dimethylallyl diphosphate (DMAPP) and adenosine 5'-diphosphate (ADP) or adenosine 5'-triphosphate (ATP) (Ihara et al. 1984; Taya et al. 1978). iP-ribotides can then be hydroxylated to tZ-ribotides by cytochrome P450 monooxygenase (CYP735A) (Takei et al. 2004). These cytokinin ribotides are converted to free-base CKs by cytokinin-activating enzymes LONELY GUYs (LOGs) (Kurakawa et al. 2007; Kuroha et al. 2009; Tokunaga et al. 2012). In addition, $\mathrm{cZ}$ and $\mathrm{tZ}$ can be enzymatically interconverted by zeatin cis-trans isomerase (Bassil et al. 1993; Sakakibara 2006). In Arabidopsis, IPT1 and IPT3 to IPT8 are involved in iP and tZ biosynthesis (Kakimoto 2001; Sun et al. 2003; Takei et al. 2001), while IPT2 and IPT9 are involved in cZ biosynthesis (Golovko et al. 2002). CYP735A1 is abundant in roots and flowers in 
Arabidopsis, while CYP735A2 specifically accumulates in roots (Takei et al. 2004). CYP735As are required for shoot growth (Kiba et al. 2013). Cytokinin oxidase/dehydrogenase (CKX) catalyses the irreversible degradation of CKs (Galuszka et al. 2001; Galuszka et al. 2007; Schmulling et al. 2003). CKXs play important roles in controlling CK levels in plant tissues. In Arabidopsis, $C K X 3$ and $C K X 5$ regulate the activity of reproductive meristems (Bartrina et al. 2011). In rice, $O_{s} C K X 4$ mediates crown root development by integrating cytokinin and auxin signalling (Gao et al. 2014).

CKs play important roles in flower bud development and floral sex differentiation (Chandler 2011; Gerashchenkov \& Rozhnova 2013; Yamasaki et al. 2005). However, the roles of CK biosynthesis genes IPTs and CYP735A and catabolism gene CKXs in Jatropha are not clear. In this study, we isolated sequences of cytokinin metabolic genes, including 6 IPTs, one $J_{c} C Y P 735 A$ and $7 J_{c} C K X s$, using the Jatropha Genome Database (Hirakawa et al. 2012; Sato et al. 2010; Wu et al. 2015). The 14 genes showed different expression patterns in different tissues of Jatropha. Some of them exhibited tissue-specific expression. JCIPT6 was only expressed in seeds a few days after pollination. $J c C Y P 735 A$ was highly expressed in roots and seeds. $J_{c} C K X 4$ was expressed mainly in seeds. In addition, CK types and contents were detected in flower buds and seeds. With flower bud development iP-type CKs increased, while tZ-type CKs decreased. With seed development, tZ-type CKs increased, while iP-type CKs decreased. The Jccyp735a mutants were obtained by the clustered regularly interspaced short palindromic repeats (CRISPR)Cas9 system. Compared with the wild-type (WT) plants, the concentrations of tZ and tZR decreased significantly in the Jccyp $735 a$ mutants, which showed severely retarded growth. These results will be helpful for future studies of the functions of these genes and for improving the biological characteristics of Jatropha.

\section{MATERIALS \& METHODS}

\section{Plant materials and growth conditions}

Three-year-old Jatropha trees were grown in the field at Xishuangbanna Tropical Botanical Garden of the Chinese Academy of Sciences, Mengla County, Yunnan Province, China (21 ${ }^{\circ} 54^{\prime}$ $\mathrm{N}, 101^{\circ} 46^{\prime} \mathrm{E} ; 580 \mathrm{~m}$ in altitude). The seedlings of WT and the T1 plants of Jccyp $735 \mathrm{a}$ mutants were grown in the greenhouse $\left(28^{\circ} \mathrm{C}, 12 \mathrm{~h}\right.$ light/ $12 \mathrm{~h}$ dark, $70 \%$ humidity). Flower buds, ovules, 
and seeds at different developmental stages were collected in May to July 2015 for qRT-PCR analysis of JcIPT6 expression and quantification of cytokinin contents. All other samples used in qRT-PCR expriments were collected at the same time in May 2015. Various plant tissue samples, including lateral roots of 1-2 $\mathrm{mm}$ in diameter with fine-roots and root tips, shoot apex of $0.3 \mathrm{~cm}$ in length from the top of shoots, stems of $1.5 \mathrm{~cm}$ in diameter, young leaf blades of 2 $\mathrm{cm}$ in length, mature leaf blades of $15 \mathrm{~cm}$ in length, flower buds of $0.3 \mathrm{~cm}$ in length, just-opened female and male flowers, fruits of 15 days after pollination, and seeds of 30 days after pollination, were harvested for qRT-PCR analysis. All tissues were immediately frozen in liquid nitrogen and stored at $-80^{\circ} \mathrm{C}$ until needed.

\section{Gene identification and isolation}

Sequences of orthologous IPT, CYP735A and CKX genes from Arabidopsis that were available in the GenBank database were used as query sequences for BLAST analysis using GenBank, the Jatropha Genome Database (http:/www.kazusa.or.jp/Jatropha/index.html) and our Jatropha transcriptome data (Chen et al. 2014; Pan et al. 2014). The full length of complementary DNA (cDNA) and genomic DNA sequences of the $J_{c I P T}, J_{c} C Y P 735 A$, and the $J_{c} C K X$ genes were obtained by PCR amplification. The PCR products were subsequently cloned into the pGEM-T vector (Promega Corporation, Madison, WI, United States) and sequenced. The GenBank accession numbers for the nucleotide sequences of these genes are listed in Table S1. Primers used in PCR are listed in Table S2.

\section{Sequence comparison and phylogenetic analysis}

Sequence chromatograms were examined and edited using Chromas Version 2.23

(http://technelysium.com.au/). Related sequences were identified with BLAST (http://www.ncbi.nlm.nih.gov/BLAST/). A phylogenetic tree was generated with MEGA 7.0 (http://www.megasoftware.net/) using the Poisson model with gamma-distributed rates and 1000 bootstrap replicates.

\section{Expression pattern analysis by qRT-PCR}

Total RNA was extracted from each tissue, and first-strand cDNA was synthesized with a PrimeScript ${ }^{\circledR}$ RT Reagent Kit with gDNA Eraser (Takara, Dalian, China) according to the manufacturer's instructions. qRT-PCR was performed with LightCycler® 480 SYBR Green I 
129 Master (Roche) on the Roche 480 Real-Time PCR Detection System (Roche Diagnostics). qRT-

130 PCR was performed with two independent biological replicates (tissue samples were harvested

131 from different plants) and three technical replicates for each sample. Data were analysed using

132 the $2^{-\triangle \Delta C T}$ method as described by Livak and Schmittgen (Livak \& Schmittgen 2001).

133 Expression levels of specific genes were normalized to that of the actin gene in Jatropha (Zhang 134 et al. 2013). Primers used in qRT-PCR are listed in Table S3.

135

136

137

138

139

140

141

142

143

144

145

146

147

148

149

150

151

152

153

154

155

156

\section{Quantification of cytokinin}

Cytokinin contents were determined by the Wuhan Greensword Creation Technology Co. Ltd., using a polymer monolith microextraction coupled with hydrophilic interaction chromatographytandem mass spectrometry method as described previously (Liu et al. 2010).

The leaves used to quantify the CKs were the third and fourth new leaves from four-month-old WT and the T1 plants of Jccyp $735 a$ mutants. Three independent biological replicates and three technical replicates were measured for each sample. The data were analyzed using the Statistical Product and Service Solutions software (SPSS Inc., Chicago, IL, USA, version 16.0). Differences among the means were determined using a one-way ANOVA with Tukey's or Tamhane's post hoc tests $(\mathrm{p}<0.05)$.

\section{Construction of CRISPR/Cas9 vectors and transformation of Jatropha}

The sequence of $J_{C} C Y P 735 A$ (GenBank accession no. XM_012222581.2) was analyzed with the online tool CRISPR-P (http://cbi.hzau.edu.cn/crispr/) to find the target sites of CRISPR/Cas9. pYLsgRNA-AtU3d/LacZ (GenBank accession no. KR029100) as the single-guide RNA (sgRNA) intermediate plasmid, and pYLCRISPR/Cas9P $35 \mathrm{~s}-\mathrm{N}$ (GenBank accession no. KR029112) as the binary vector were used for the CRISPR-Cas9 construction of $J_{c} C Y P 735 \mathrm{~A}$ following the instruction of the CRISPR-Cas9 System (Ma et al. 2015). Transformation of Jatropha with Agrobacterium strain EHA105 carrying the JCCYP735A CRISPR/Cas9 construction was performed according to the protocol described by $\mathrm{Fu}$ et al. (2015). The Jccyp 735 a mutants in transgenic Jatropha plants were identified by PCR amplification and DNA sequencing using a pair of primers, XB619 (5'-ATGGCCATGATATTAACAACTCTATTAG3') and XB620 (5'-GCGGTTCTATCCCATTCCAGTATAT-3'). 
157

\section{RESULTS}

\section{Cloning and identification of JcIPTs, JcCYP735A, and JcCKXs}

Using all annotated Arabidopsis IPT, CYP735A and CKX family members in the TAIR as query sequences to perform a BLAST analysis in GenBank and with our Jatropha transcriptome data (Chen et al. 2014; Pan et al. 2014), we identified and cloned IPT, CYP735A and CKX orthologous sequences in Jatropha. The Jatropha IPT family included only six members, while there are nine members in Arabidopsis. These genes were named JcIPT1, JcIPT2, JcIPT3, JcIPT5, JcIPT6, and JCIPT9. The BLAST analysis identified only one member of the CYP735A family, $J_{c} C Y P 735 A$. The $C K X$ gene family, encoding degradation enzymes, included the same seven members in Jatropha as in Arabidopsis. These genes were named $J_{c} C K X 1, J_{c} C K X 2$, $J_{c} C K X 3, J_{c} C K X 4, J_{c} C K X 5, J_{c} C K X 6$, and $J_{c} C K X 7$.

Sequence structure analysis showed that $I P T, C Y P 735 A$, and $C K X$ family members shared almost the same numbers of exons and introns between Jatropha and Arabidopsis and had similar exon lengths (Figure 2, 3). JcIPT6 has two more exons than IPT6 from Arabidopsis. However, the extra two exon sequences are short and are not part of the P-loop NTPase domain (Figure 2a).

\section{Phylogenetic analysis of JcIPTs, JcCYP735A, and JcCKXs}

To analyse the phylogenetic relationships between orthologous genes, phylogenetic analysis were performed. IPT, CYP735A and CKX family members from Arabidopsis thaliana, Ricinus communis, and Oryza sativa were compared with those from Jatropha. Orthologues of IPT1, 2, 3, 5, and 7 formed a clade, while IPT9 formed a single clade (Figure 4a). JcCYP735A along with other dicotyledon CYP735As formed a clade, while CYP735A3 and 4 of Oryza sativa formed another clade (Figure 4b). Orthologues of CKX1, 5, 6, and 7 formed a clade, while those of CKX2, 3, and 4 formed a separate clade (Figure 5). These results showed that JcIPTs, JcCYP735A, and JcCKXs were most closely related to genes from Ricinus communis, which also belongs to the Euphorbiaceae family.

\section{Expression patterns of $J c I P T s, J c C Y P 735 A$, and $J c C K X s$ in different tissues}

In order to gain more information of these gene family members in Jatropha, the temporal and spatial expression patterns of these genes were analysed using qRT-PCR. JCIPT1 was mainly expressed in roots (Figure 6a). JcIPT2 was mainly expressed in roots, shoot apical meristems, 
186

187

188

189

mature leaves, and flower buds (Figure 6b). JcIPT3 showed much higher expression levels in stems and mature leaves than other tissues (Figure 6c). JCIPT5 exhibited high expression levels in roots and mature leaves (Figure 6d). JcIPT9 only showed high expression levels in mature leaves (Figure 6f). The expression of JcIPT6 was not detected in most of the plant tissues indicated above. After analysing more tissues (Figure S1), we found that JcIPT6 began to be expressed in seeds a few days after fertilization, with the strongest expression observed in seeds at 10 days after fertilization; the expression levels then decreased rapidly. In seeds at 20 days after fertilization, JcIPT6 expression decreased by a factor of 5 compared with seeds at 10 days after fertilization (Figure 6e). The expression levels of $J C C Y P 735 A$ were higher in roots, flower buds, and seeds than other tissues (Figure7a). $J c C K X 1$ was mainly expressed in flower buds, roots, and female flowers (Figure $7 \mathrm{~b}$ ). $J c C K X 2$ showed very strong expression in female flowers and seeds (Figure 7c). $J_{c} C K X 3$ was highly expressed in male flowers (Figure 7d). $J c C K X 4$ exhibited high expression levels in mature leaves and extremely high expression in seeds (Figure 7e). $J c C K X 5$ was mainly expressed in stems, young leaves, and fruit (Figure 7f). JcCKX6 was expressed in all tissues (Figure $7 \mathrm{~g}$ ). $J c C K X 7$ was mainly expressed in roots (Figure $7 \mathrm{~h}$ ).

\section{Endogenous CK contents in flower buds and seeds}

In order to learn more about the distribution of endogenous cytokinins, we measured the contents of endogenous CKs in Jatropha flower buds and seeds at different developmental stages (Figure S1). Different profiles were observed for each CK variant with flower bud and seed development in Jatropha (Table 1). The contents of iP and its variant iPR increased approximately 177-fold and 9-fold, respectively, from the flower bud 1 (FB1) stage to the flower bud 2 (FB2) stage. Compared with the FB1 stage, tZ content was approximately doubled in the FB2 stage, while tZR content was reduced by $93 \%$, resulting in a decrease in the amount of total active $\mathrm{tZ}$ variants. Conversely, the contents of $\mathrm{tZ}$ variants increased remarkably during seed development; compared with ovules, the tZ content increased 187-fold and the tZR content increased 496-fold in seeds at 20 DAP.

\section{Jccyp735a mutants generated by CRISPR-Cas9 system showed retarded growth}

To explore the biological function of JcCYP735A in Jatropha, we generated Jatropha transformants with $J_{c} C Y P 735 A$ knocked out using the CRISPR-Cas9 system (Figure 8). Three homozygous mutant lines, L1, L2, and L3, were obtained by DNA sequencing (Figure 8a). 
216 Endogenous contents of CKs in the leaves of two lines of Jccyp 735 a mutants (L2 and L3) and

217 WT plants were examined. The results showed that the concentrations of $\mathrm{tZ}$ and $\mathrm{tZR}$, and $\mathrm{cZ}$ and

218 cZR significantly decreased, whereas the concentrations of iP and iPR significantly increased in

$219 J c c y p 735 a$ mutants compared with that of the WT (Figure 8b). Jccyp735a mutants showed

220 severely retarded growth, and the mutant plants were only approximately a quarter the height of

221 the WT plants (Figure 8c and d).

\section{DISCUSSION}

223

224

225

226

227

228

229

230

231

232

233

234

235

236

237

238

239

240

241

242

243

244

245

In our study, six IPT family members were identified in Jatropha. The number of IPT genes differs among plant species; for example, there are six IPTs in Ricinus communis, while there are nine IPTs in both Arabidopsis (Kakimoto 2001; Takei et al. 2001) and rice (Figure 4a). JcIPTs have the same number of exons as those in Arabidopsis, except JcIPT6, which has two more exons than AtIPT6. The third exon of JCIPT6 has almost the same number of base pairs as the only exon in AtIPT6 (Figure 2a). It appears that the other two small exons were lost during evolution. Expression pattern analysis revealed that different JcIPT members are expressed in different tissues in Jatropha (Figure 6). JCIPT1 had an expression pattern similar to that of AtIPT1 and was mostly expressed in roots, shoot apical meristems, and seeds (Miyawaki et al. 2004). JcIPT2 and JcIPT9 were assigned to the same cluster as their orthologues (Figure 4a). In Arabidopsis, AtIPT2 and AtIPT9 are expressed ubiquitously, with stronger expression in proliferating tissues, including the root and shoot apical meristems and leaf primordia (Miyawaki et al. 2004). Similarly, JcIPT2 and JCIPT9 were expressed ubiquitously in Jatropha. However, the strongest expression of both was in mature leaves instead of meristems and young leaves (Figure 6b, f). Considering that AtIPT2 and AtIPT9 are tRNA-IPTs, which only catalyse cZ biosynthesis (Golovko et al. 2002), cZ might be produced mainly in mature leaves of Jatropha. AtIPT3 was found to be responsible for nitrate-dependent cytokinin biosynthesis and is predominantly expressed in the phloem (Miyawaki et al. 2004; Takei et al. 2002). In our study, JCIPT3 was strongly expressed in stems and mature leaves, which contain abundant phloem (Figure 6c). A study by Miyawaki et al. (2004) showed that GUS activity is not detected in Arabidopsis transformants carrying AtIPT6::GUS. RT-PCR analysis indicated that it was abundant in siliques. Analogously, we did not detect JcIPT6 in most tissues of Jatropha, including fruits. We found that JcIPT6 was mainly expressed in seeds at 10 days after 
246 pollination, and its expression decreased rapidly thereafter (Figure 6e). It is possible that the 247 previous study missed this critical phase in the seeds chosen for GUS staining (Miyawaki et al. 2004). Our results suggested that different $J_{c} I P T$ family members play different roles in the development of Jatropha and that some JCIPT members could be used to cultivate high-yield varieties of Jatropha using transgenic technology.

251

Although none of the ATP/ADP JcIPTs were found to be highly expressed in flowers, iP, iPR, and $\mathrm{tZ}$ contents increased with the development of flower buds (Table 1). It is known that CKs can be transported through the plant vascular system (Hirose et al. 2007). JcCYP735A was found to be highly expressed in flower buds (Figure 7a). iP-type CKs may be transported into flowers, and some may then be used to generate tZ-type CKs via JcCYP735A. In addition, it has been reported that the $\mathrm{tZ}$ concentration is up-regulated in the early development of tomato fruits (Matsuo et al. 2012). Our study showed that $J c C Y P 735 A$ was highly expressed and that the $t Z$ concentration increased with seed development in Jatropha (Table 1). Thus, JcCYP735A might play important roles in seed development by controlling tZ biosynthesis. Similar to Ricinus communis (Chan et al. 2010), only one CYP735A gene was found in Jatropha, although there are two CYP735A members in Arabidopsis and rice (Takei et al. 2004; Tsai et al. 2012).

Unlike $J_{C I P T}$ or $J_{C} C Y P 735 A$ family, the $J_{C} C K X$ family in Jatropha has the same number of members as that in Arabidopsis (Figure 3). In addition, the $J c C K X$ and AtCKX orthologues contain the same number of exons, with 5 exons in $C K X 1-6$ and 4 exons in $C K X 7$ (Figure 3). Further expression analysis showed that $J c C K X 2$ was mostly expressed in female flowers, whereas $J_{C} C K X 3$ was mostly expressed in male flowers (Figure 7c, d). Both $J c C K X 2$ and $J c C K X 4$ were expressed strongly in seeds (Figure 7c, e). These tissue-specific expression genes may be chosen to adjust the CK content in these tissues using transgenic methods. It has been reported that reduced expression of $\mathrm{OsCKX}_{\mathrm{S}}$ causes cytokinin accumulation in inflorescence meristems and increases the number of reproductive organs, resulting in enhanced grain yield (Ashikari et al. 2005). Decreased expression of $C K X$ orthologues may also lead to increased yield in Jatropha. Moreover, overexpression of some $C K X$ members can also improve resistance. Overexpression of $C K X 1$ or $C K X 2$ in Arabidopsis and other species causes elongation of the primary root and increases root branching (Galuszka et al. 2004; Mrízová et al. 2013; Pospisilova et al. 2016; Werner et al. 2001; Yang et al. 2003), while overexpression of AtCKX7 results in an 
276

277

278

279

280

281

282

283

284

285

286

287

288

289

290

291

292

293

294

295

296

297

298

299

300

301

302

303

304

305

opposite phenotype (Kollmer et al. 2014). Specific expression of $J c C K X 1$ or $J c C K X 2$ in roots might be used to transform a shallow root system into a deep root system to improve the growth and lodging resistance of Jatropha. Furthermore, root system development might enhance tolerance to drought stress. Remarkably, $J_{c} C K X 4$ expression was much higher in seeds than other tissues (Figure 7e), suggesting that JcCKX4 may be a key enzyme regulating cytokinin levels to affect seed development.

In early flower bud development, the content of iP-type CKs increased significantly, while that of tZ-type CKs decreased. This result indicated that iP-type CKs participate more in early flower bud development than tZ-type CKs. In tomato, iP-type CK contents decrease during fruit ripening (Matsuo et al. 2012). By contrast, in early seed development, the content of tZ-type CKs increased substantially, while that of iP-type CKs decreased. This result, which is in accordance with high expression of $J_{c} C Y P 735 \mathrm{~A}$ in seeds, suggested that tZ-type CKs are dominant in early seed development. Many differences were observed in CK contents in different periods of Jatropha flower bud and seed development. Our results indicate that iP-type CKs can be used to improve the number of flowers in Jatropha, while tZ-type CKs can be used to enlarge seeds.

The single cyp735a1 or cyp735a2 mutant Arabidopsis showed slight decreases in tZ and tZR concentration compared with that of WT, while the double mutants showed great decreases (Kiba et al. 2013). In addition, cyp $735 a 1$ cyp $735 a 2$ double mutants exhibited retarded shoot growth (Kiba et al. 2013). Similarly, in this study, Jccyp $735 a$ mutants showed substantial decreases in $\mathrm{tZ}$ and $\mathrm{tZR}$ concentrations (Figure $8 \mathrm{~b}$ ), which is consistent with that only a single member of JcCYP735A was found in Jatropha genome (Wu et al. 2015). We noticed, however, tZ and tZR did not completely disappear in Jccyp $735 a$ mutants (Figure 8b), which may result from conversion of $\mathrm{cZ}$ and $\mathrm{cZR}$. This notion is supported by the fact that the concentrations of $\mathrm{cZ}$ and cZR also significantly decreased in Jccyp $735 a$ mutants (Figure 8b), and that cis-trans isomerase activity for interconversion between cZ-type and tZ-type CKs has been reported in several plant species (Bassil et al. 1993; Kudo et al. 2012; Suttle \& Banowetz 2000).

\section{CONCLUSIONS}

In this study, we isolated the members of the $J_{c I P T}, J_{c} C Y P 735 A$ and $J_{c} C K X$ gene families and analysed their temporal and spatial expression patterns. Different family members exhibited different expression patterns. Different types of CKs seemed to influence the development of 
306 flower buds and seeds, respectively. The analysis of the Jccyp $735 a$ mutants revealed that

$307 J_{c} C Y P 735 A$ plays an important role in $\mathrm{tZ}$ biosynthesis in Jatropha. These results will be helpful

308 for further function studies of cytokinin metabolic genes and improving agronomic

309 characteristics of Jatropha by genetic engineering of cytokinin metabolism.

\section{ACKNOWLEDGMENTS}

311 We thank Prof. Yao-Guang Liu (South China Agricultural University, China) for providing vectors

312 pYLCRISPR/Cas9P ${ }_{35 \mathrm{~s}} \mathrm{-N}$ and pYLsgRNA-AtU3d. The authors gratefully acknowledge the Central Laboratory of

313 the Xishuangbanna Tropical Botanical Garden for providing research facilities.

\section{Abbreviations}

315 BLAST basic local alignment search tool

316 CK cytokinin

317 CKX cytokinin oxidase/dehydrogenase

318 CRISPR clustered regularly interspaced short palindromic repeats

319 CYP735A cytochrome P450 monooxygenase, family 735, subfamily A

320 cZ cis-zeatin

321 DZ dihydrozeatin

322 DZR dihydrozeatin-riboside

323 iP N6-( $\Delta 2$ - isopentenyl)-adenine

324 IP9G N6-( $\Delta 2$ - isopentenyl)-adenine-9-glucoside

325 iPR iP-riboside

326 IPT adenosine phosphate-isopentenyltransferases

327 LOG LONELY GUY

328 qRT-PCR quantitative reverse transcriptase-polymerase chain reaction

329 tZ trans-zeatin

330 tZ9G trans-zeatin-9-glucoside

331 tZR tZ-riboside

\section{REFERENCES}

Akashi K. 2012. Jatropha research: A new frontier for biofuel development. Plant Biotechnol 29:121. 10.5511/plantbiotechnology.12.0003p

Ashikari M, Sakakibara H, Lin S, Yamamoto T, Takashi T, Nishimura A, Angeles ER, Qian Q, Kitano H, and Matsuoka M. 2005. Cytokinin oxidase regulates rice grain production. Science 309:741-745.

Bartrina I, Otto E, Strnad M, Werner T, and Schmulling T. 2011. Cytokinin regulates the activity of reproductive meristems, flower organ size, ovule formation, and thus seed yield in Arabidopsis thaliana. Plant Cell 
23:69-80. 10.1105/tpc.110.079079

Bassil NV, Mok DW, and Mok MC. 1993. Partial purification of a cis-trans-isomerase of zeatin from immature seed of Phaseolus vulgaris L. Plant Physiol 102:867-872.

Chan AP, Crabtree J, Zhao Q, Lorenzi H, Orvis J, Puiu D, Melake-Berhan A, Jones KM, Redman J, and Chen G. 2010. Draft genome sequence of the oilseed species Ricinus communis. Nat Biotechnol 28:951.

Chandler J. 2011. The hormonal regulation of flower development. J Plant Growth Regul 30:242-254.

Chen M-S, Pan B-Z, Wang G-J, Ni J, Niu L, and Xu Z-F. 2014. Analysis of the transcriptional responses in inflorescence buds of Jatropha curcas exposed to cytokinin treatment. BMC Plant Biol 14:318.

Francis G, Edinger R, and Becker K. 2005. A concept for simultaneous wasteland reclamation, fuel production, and socio - economic development in degraded areas in India: Need, potential and perspectives of Jatropha plantations. Natural Resources Forum: Wiley Online Library. p 12-24.

Fröschle M, Horn H, and Spring O. 2017. Effects of the cytokinins 6-benzyladenine and forchlorfenuron on fruit-, seed-and yield parameters according to developmental stages of flowers of the biofuel plant Jatropha curcas L.(Euphorbiaceae). Plant Growth Regul 81:293-303.

Fu Q, Li C, Tang M, Tao Y-B, Pan B-Z, Zhang L, Niu L, He H, Wang X, and Xu Z-F. 2015. An efficient protocol for Agrobacterium-mediated transformation of the biofuel plant Jatropha curcas by optimizing kanamycin concentration and duration of delayed selection. Plant Biotechnol Rep 9:405-416.

Galuszka P, Frébort I, Šebela M, Sauer P, Jacobsen S, and Peč P. 2001. Cytokinin oxidase or dehydrogenase? FEBS J 268:450-461.

Galuszka P, Frebortova J, Werner T, Yamada M, Strnad M, Schmulling T, and Frebort I. 2004. Cytokinin oxidase/dehydrogenase genes in barley and wheat: cloning and heterologous expression. FEBS J 271:39904002. 10.1111/j.1432-1033.2004.04334.x

Galuszka P, Popelková H, Werner T, Frébortová J, Pospíšilová H, Mik V, Köllmer I, Schmülling T, and Frébort I. 2007. Biochemical characterization of cytokinin oxidases/dehydrogenases from Arabidopsis thaliana expressed in Nicotiana tabacum L. Journal of Plant Growth Regulation 26:255-267.

Gan S, and Amasino RM. 1995. Inhibition of leaf senescence by autoregulated production of cytokinin. Science 270:1986-1988.

Gao S, Fang J, Xu F, Wang W, Sun X, Chu J, Cai B, Feng Y, and Chu C. 2014. CYTOKININ OXIDASE/DEHYDROGENASE4 Integrates Cytokinin and Auxin Signaling to Control Rice Crown Root Formation. Plant Physiol 165:1035-1046. 10.1104/pp.114.238584

Gerashchenkov G, and Rozhnova N. 2013. The involvement of phytohormones in the plant sex regulation. Russ $J$ Plant Physiol 60:597-610.

Golovko A, Sitbon F, Tillberg E, and Nicander B. 2002. Identification of a tRNA isopentenyltransferase gene from Arabidopsis thaliana. Plant Mol Biol 49:161-169.

Hirakawa H, Tsuchimoto S, Sakai H, Nakayama S, Fujishiro T, Kishida Y, Kohara M, Watanabe A, Yamada M, and Aizu T. 2012. Upgraded genomic information of Jatropha curcas L. Plant Biotechnol 29:123-130.

Hirose N, Takei K, Kuroha T, Kamada-Nobusada T, Hayashi H, and Sakakibara H. 2007. Regulation of cytokinin biosynthesis, compartmentalization and translocation. $J$ Exper Bot 59:75-83.

Ihara M, Taya Y, Nishimura S, and Tanaka Y. 1984. Purification and some properties of $42_{-}$ isopentenylpyrophosphate: $5^{\prime} \mathrm{Amp} \Delta^{2}$-isopentenyltransferase from the cellular slime mold Dictyostelium discoideum. Arch Biochem Biophys 230:652-660. 
381

382

383

384

385

386

387

388

389

390

391

392

393

394

395

396

397

398

399

400

401

402

403

404

405

406

407

408

409

410

411

412

413

414

415

416

417

418

419

420

421

Kakimoto T. 2001. Identification of plant cytokinin biosynthetic enzymes as dimethylallyl diphosphate: ATP/ADP isopentenyltransferases. Plant Cell Physiol 42:677-685.

Kiba T, Takei K, Kojima M, and Sakakibara H. 2013. Side-chain modification of cytokinins controls shoot growth in Arabidopsis. Dev Cell 27:452-461. 10.1016/j.devcel.2013.10.004

Kollmer I, Novak O, Strnad M, Schmulling T, and Werner T. 2014. Overexpression of the cytosolic cytokinin oxidase/dehydrogenase (CKX7) from Arabidopsis causes specific changes in root growth and xylem differentiation. Plant J: for Cell and Molecular Biology 78:359-371. 10.1111/tpj.12477

Kudo T, Kiba T, and Sakakibara H. 2010. Metabolism and long-distance translocation of cytokinins. $J$ Integr Plant Biol 52:53-60. 10.1111/j.1744-7909.2010.00898.x

Kudo T, Makita N, Kojima M, Tokunaga H, and Sakakibara H. 2012. Cytokinin activity of cis-zeatin and phenotypic alterations induced by overexpression of putative cis-zeatin- $O$-glucosyltransferase in rice. Plant Physiol 160:319-331. 10.1104/pp.112.196733

Kumar A, and Sharma S. 2008. An evaluation of multipurpose oil seed crop for industrial uses (Jatropha curcas L.): A review. Ind Crops Prod 28:1-10. 10.1016/j.indcrop.2008.01.001

Kumar Tiwari A, Kumar A, and Raheman H. 2007. Biodiesel production from jatropha oil (Jatropha curcas) with high free fatty acids: An optimized process. Biomass and Bioenerg 31:569-575. 10.1016/j.biombioe.2007.03.003

Kurakawa T, Ueda N, Maekawa M, Kobayashi K, Kojima M, Nagato Y, Sakakibara H, and Kyozuka J. 2007. Direct control of shoot meristem activity by a cytokinin-activating enzyme. Nature 445:652-655. 10.1038/nature05504

Kuroha T, Tokunaga H, Kojima M, Ueda N, Ishida T, Nagawa S, Fukuda H, Sugimoto K, and Sakakibara H. 2009. Functional analyses of LONELY GUY cytokinin-activating enzymes reveal the importance of the direct activation pathway in Arabidopsis. Plant Cell 21:3152-3169. 10.1105/tpc.109.068676

Liu Z, Wei F, and Feng Y-Q. 2010. Determination of cytokinins in plant samples by polymer monolith microextraction coupled with hydrophilic interaction chromatography-tandem mass spectrometry. Anal Methods 2:1676-1685.

Livak KJ, and Schmittgen TD. 2001. Analysis of relative gene expression data using real-time quantitative PCR and the $2^{-\triangle \Delta C T}$ method. Methods 25:402-408.

Ma X, Zhang Q, Zhu Q, Liu W, Chen Y, Qiu R, Wang B, Yang Z, Li H, and Lin Y. 2015. A robust CRISPR/Cas9 system for convenient, high-efficiency multiplex genome editing in monocot and dicot plants. Mol Plant 8:1274-1284.

Makkar HP, and Becker K. 2009. Jatropha curcas, a promising crop for the generation of biodiesel and value - added coproducts. Eur J Lipid Sci Technol 111:773-787.

Matsuo S, Kikuchi K, Fukuda M, Honda I, and Imanishi S. 2012. Roles and regulation of cytokinins in tomato fruit development. J Exper Bot 63:5569-5579.

Miyawaki K, Matsumoto-Kitano M, and Kakimoto T. 2004. Expression of cytokinin biosynthetic isopentenyltransferase genes in Arabidopsis: tissue specificity and regulation by auxin, cytokinin, and nitrate. Plant J 37:128-138.

Mok DW, and Mok MC. 2001. Cytokinin metabolism and action. Annu Rev Plant Biol 52:89-118.

Mrízová K, Jiskrová E, Vyroubalová Š, Novák O, Ohnoutková L, Pospíšilová H, Frébort I, Harwood WA, and Galuszka P. 2013. Overexpression of cytokinin dehydrogenase genes in barley (Hordeum vulgare cv.

PeerJ reviewing PDF | (2018:02:25677:1:1:NEW 16 Apr 2018) 
Golden Promise) fundamentally affects morphology and fertility. PLoS One 8:e79029.

Pan B-Z, Chen M-S, Ni J, and Xu Z-F. 2014. Transcriptome of the inflorescence meristems of the biofuel plant Jatropha curcas treated with cytokinin. BMC genomics 15:974. 10.1186/1471-2164-15-974

Pan B-Z, and Xu Z-F. 2011. Benzyladenine treatment significantly increases the seed yield of the biofuel plant Jatropha curcas. Plant Growth Regul 30:166-174.

Pospisilova H, Jiskrova E, Vojta P, Mrizova K, Kokas F, Cudejkova MM, Bergougnoux V, Plihal O, Klimesova J, Novak O, Dzurova L, Frebort I, and Galuszka P. 2016. Transgenic barley overexpressing a cytokinin dehydrogenase gene shows greater tolerance to drought stress. New Biotechnol 33:692-705. 10.1016/j.nbt.2015.12.005

Rao GR, Korwar GR, Shanker AK, and Ramakrishna YS. 2008. Genetic associations, variability and diversity in seed characters, growth, reproductive phenology and yield in Jatropha curcas (L.) accessions. Trees 22:697-709. 10.1007/s00468-008-0229-4

Sakakibara H. 2006. Cytokinins: activity, biosynthesis, and translocation. Annu Rev Plant Biol 57:431-449.

Sato S, Hirakawa H, Isobe S, Fukai E, Watanabe A, Kato M, Kawashima K, Minami C, Muraki A, and Nakazaki N. 2010. Sequence analysis of the genome of an oil-bearing tree, Jatropha curcas L. DNA Res 18:65-76.

Schmulling T, Werner T, Riefler M, Krupkova E, and Bartrina y Manns I. 2003. Structure and function of cytokinin oxidase/dehydrogenase genes of maize, rice, Arabidopsis and other species. $J$ Plant Res 116:241-252. 10.1007/s10265-003-0096-4

Shimizu-Sato S, Tanaka M, and Mori H. 2009. Auxin-cytokinin interactions in the control of shoot branching. Plant Mol Biol 69:429.

Sun J, Niu Q-W, Tarkowski P, Zheng B, Tarkowska D, Sandberg G, Chua N-H, and Zuo J. 2003. The Arabidopsis AtIPT8/PGA22 gene encodes an isopentenyl transferase that is involved in de novo cytokinin biosynthesis. Plant Physiol 131:167-176.

Suttle JC, and Banowetz G. 2000. Changes in cis - zeatin and cis - zeatin riboside levels and biological activity during potato tuber dormancy. Physiologia Plantarum 109:68-74.

Takei K, Sakakibara H, and Sugiyama T. 2001. Identification of genes encoding adenylate isopentenyltransferase, a cytokinin biosynthesis enzyme, in Arabidopsis thaliana. $J$ Biol Chem 276:26405-26410. 10.1074/jbc.M102130200

Takei K, Takahashi T, Sugiyama T, Yamaya T, and Sakakibara H. 2002. Multiple routes communicating nitrogen availability from roots to shoots: a signal transduction pathway mediated by cytokinin. J Exper Bot 53:971977.

Takei K, Yamaya T, and Sakakibara H. 2004. Arabidopsis CYP735A1 and CYP735A2 encode cytokinin hydroxylases that catalyze the biosynthesis of trans-Zeatin. $J$ Biol Chem 279:41866-41872. 10.1074/jbc.M406337200

Tanaka M, Takei K, Kojima M, Sakakibara H, and Mori H. 2006. Auxin controls local cytokinin biosynthesis in the nodal stem in apical dominance. Plant $J$ 45:1028-1036.

Taya Y, Tanaka Y, and Nishimura S. 1978. 5'-AMP is a direct precursor of cytokinin in Dictyostelium discoideum. Nature 271:545-547.

Tokunaga H, Kojima M, Kuroha T, Ishida T, Sugimoto K, Kiba T, and Sakakibara H. 2012. Arabidopsis lonely guy (LOG) multiple mutants reveal a central role of the LOG - dependent pathway in cytokinin activation. Plant $J$ 69:355-365. 
Tsai Y-C, Weir NR, Hill K, Zhang W, Kim HJ, Shiu S-H, Schaller GE, and Kieber JJ. 2012. Characterization of genes involved in cytokinin signaling and metabolism from rice. Plant Physiol 158:1666-1684.

Werner T, Motyka V, Laucou V, Smets R, Van Onckelen H, and Schmulling T. 2003. Cytokinin-deficient transgenic Arabidopsis plants show multiple developmental alterations indicating opposite functions of cytokinins in the regulation of shoot and root meristem activity. Plant Cell 15:2532-2550. $10.1105 /$ tpc. 014928

Werner T, Motyka V, Strnad M, and Schmulling T. 2001. Regulation of plant growth by cytokinin. Proc Natl Acad Sci U S A 98:10487-10492. 10.1073/pnas.171304098

Wu P, Zhou C, Cheng S, Wu Z, Lu W, Han J, Chen Y, Chen Y, Ni P, Wang Y, Xu X, Huang Y, Song C, Wang Z, Shi N, Zhang X, Fang X, Yang Q, Jiang H, Chen Y, Li M, Wang Y, Chen F, Wang J, and Wu G. 2015. Integrated genome sequence and linkage map of physic nut (Jatropha curcas L.), a biodiesel plant. Plant Journal 81:810-821. 10.1111/tpj.12761

Yamasaki S, Fujii N, and Takahashi H. 2005. Hormonal regulation of sex expression in plants. Vitam Horm 72:79110.

Yang S, Yu H, Xu Y, and Goh CJ. 2003. Investigation of cytokinin - deficient phenotypes in Arabidopsis by ectopic expression of orchid DSCKX1. FEBS Lett 555:291-296. 10.1016/s0014-5793(03)01259-6

Zhang L, He L-L, Fu Q-T, and Xu Z-F. 2013. Selection of reliable reference genes for gene expression studies in the biofuel plant Jatropha curcas using real-time quantitative PCR. International Journal of Molecular Sciences 14:24338-24354. 10.3390/ijms141224338

\section{Figure legends}

Figure 1 Basic scheme for the cytokinin biosynthesis and degradation pathways. Solid arrows indicate pathways with genes that are known, and dotted arrows indicate pathways with genes that remain to be identified. The enzymes are marked by red frames. The iP, $\mathrm{Z}$ and their ribosides inside the dotted boxes could be degraded by CKX. cZ, cis-zeatin; DMAPP, dimethylallyldiphosphate; CKX, cytokinin oxidase/dehydrogenase; cZ, cis-zeatin; DMAPP, dimethylallyl diphosphate; iP, N6-( $\Delta 2-$ isopentenyl)adenine; IPT, adenosine phosphateisopentenyltransferase; LOG, LONELY GUY; tRNA-IPT, tRNA-isopentenyltransferase; tZ, trans-zeatin; Z, zeatin. This figure was modified and redrawn from reference (Kudo et al. 2010).

Figure 2 Genomic organization of $I P T$ and $C Y P 735 A$ family members in Jatropha and Arabidopsis. (a) IPT family members; (b) CYP735A family members. At, Arabidopsis thaliana; Jc, Jatropha curcas.

Figure 3 Genomic organization of $\boldsymbol{C K X}$ family members in Jatropha and Arabidopsis. At, Arabidopsis thaliana; Jc, Jatropha curcas. 
497

498

499

500

501

502

503

504

505

506

507

508

509

510

511

512

513

514

515

516

517

518

519

520

521

522

523

524

525

526

527

528

529

530 Supplemental Figures

Figure 4 Neighbour-joining phylogenetic tree for IPT and CYP735A family members in various species.

(a) IPT family members; (b) CYP735A family members. At, Arabidopsis thaliana; Jc, Jatropha curcas; Os, Oryza sativa; Rc, Ricinus communis.

Figure 5 Neighbour-joining phylogenetic tree for CKX family members in various species. At, Arabidopsis thaliana; Jc, Jatropha curcas; Os, Oryza sativa; Rc, Ricinus communis.

Figure 6 Expression of JcIPTs in various Jatropha tissues. (a) to (f) are expression patterns of JCIPT1, JCIPT2, JCIPT3, JCIPT5, JCIPT6, and JCIPT9, respectively. The qRT-PCR results were obtained from two independent biological replicates and three technical replicates for each sample. R, roots; SAM, shoot apical meristems; ST, stems; YL, young leaves; ML, mature leaves; FB, flower buds; FF, female flowers; MF, male flowers; FRU, fruits; SD, seeds; 0 DAP, unfertilized ovules; 10 DAP, seeds at 10 days after pollination; 20 DAP, seeds at 20 days after pollination; 30 DAP, seeds at 30 days after pollination; DAP, days after pollination.

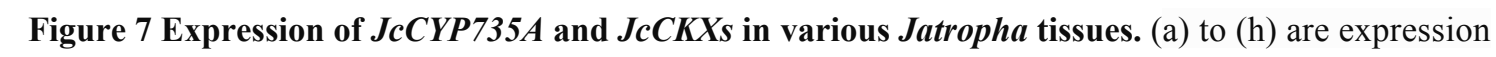
patterns of $J_{C} C Y P 735 A, J_{C} C K X 1, J_{c} C K X 2, J_{c} C K X 3, J_{c} C K X 4, J_{c} C K X 5, J_{c} C K X 6$, and $J_{C} C K X 7$, respectively. Values in the Y-axis of (e) are displayed in scientific notation. e, exponent. The qRT-PCR results were obtained from two independent biological replicates and three technical replicates for each sample. R, roots; SAM, shoot apical meristems; ST, stems; YL, young leaves; ML, mature leaves; FB, flower buds; FF, female flowers; MF, male flowers; FRU, fruits; SD, seeds.

\section{Figure 8 Generation and phenotypic variation of Jccyp $735 a$ mutants of Jatropha obtained by the} CRISPR-Cas9 system. (a) Different types of $J_{c} C Y P 735 A$ mutation generated by the CRISPR-Cas9mediated gene silencing in the progenies (T1 generation) of the three transgenic lines L1, L2, and L3. The blue characters show the selected target site sequences. The characters in purple box show the protospacer adjacent motif (PAM) sequences. The red character indicates the nucleotide insertion. WT, wild-type. (b-d) Cytokinin concentrations (b), the heights (c), and appearance (d) of the four-month-old seedlings (T1) of Jccyp 735 a mutant lines and WT. Error bars represent the standard deviation (SD) of three (b) or five (c) biological replicates. Asterisks indicate statistically significant differences compared with WT $(p<0.05)$. In (d), bar $=5 \mathrm{~cm}$.

Additional files 
531 Figure S1 Flower buds, ovules and seeds of Jatropha used in this study. A, Flower buds from the FB1 stage.

532 Bar $=1 \mathrm{~cm}$. B, Flower buds from the FB2 stage. Bar $=1 \mathrm{~cm}$. C, Unfertilized Jatropha pistil, from which the 533 ovule was obtained. Bar $=1 \mathrm{~mm}$. D, Morphology of unfertilized ovules. Bar $=1 \mathrm{~mm}$. E, Morphology of fruits 534 and seeds. The first row contains fruits at different developmental periods. The second row shows the seeds of 535 the corresponding fruits. Bar $=1 \mathrm{~cm} .0$ DAP, unfertilized ovules; 10 DAP, seeds at 10 days after pollination; 53620 DAP, seeds at 20 days after pollination; 30 DAP, seeds at 30 days after pollination; DAP, days after 537 pollination. 


\section{Figure 1}

Basic scheme for the cytokinin biosynthesis and degradation pathways.

Solid arrows indicate pathways with genes that are known, and dotted arrows indicate pathways with genes that remain to be identified. The enzymes are marked by red frames. The iP, $\mathrm{Z}$ and their ribosides inside the dotted boxes could be degraded by CKX. CZ, ciszeatin; DMAPP, dimethylallyldiphosphate; CKX, cytokinin oxidase/dehydrogenase; cZ, ciszeatin; DMAPP, dimethylallyl diphosphate; iP, N6-( $\Delta 2$ - isopentenyl)adenine; IPT, adenosine phosphate-isopentenyltransferase; LOG, LONELY GUY; tRNA-IPT, tRNAisopentenyltransferase; tZ, trans-zeatin; Z, zeatin. This figure was modified and redrawn from reference (Kudo et al. 2010).

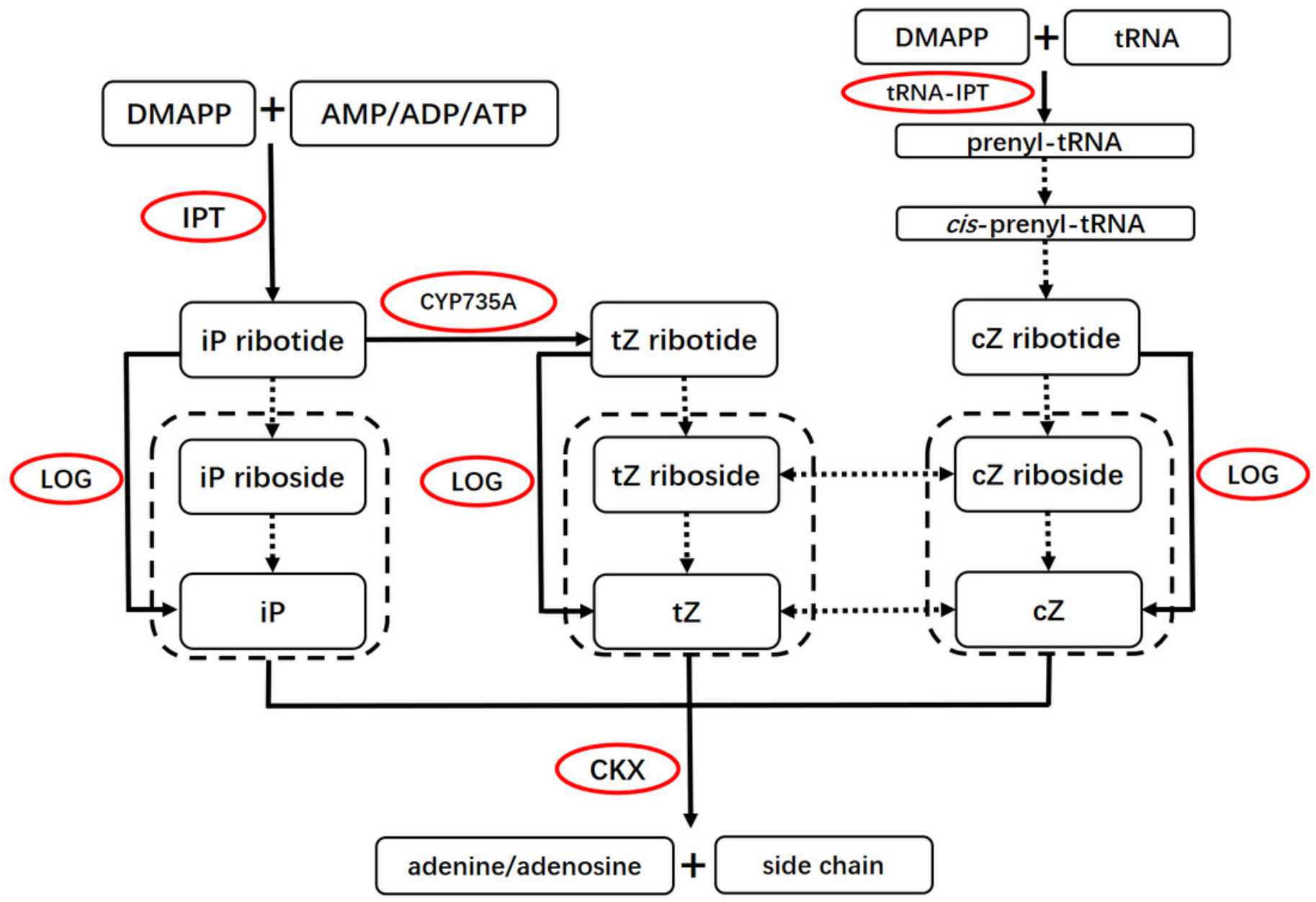


Figure 2

Genomic organization of IPT and CYP735A family members in Jatropha and Arabidopsis.

(a) IPT family members; (b) CYP735A family members. At, Arabidopsis thaliana; Jc, Jatropha curcas.

a

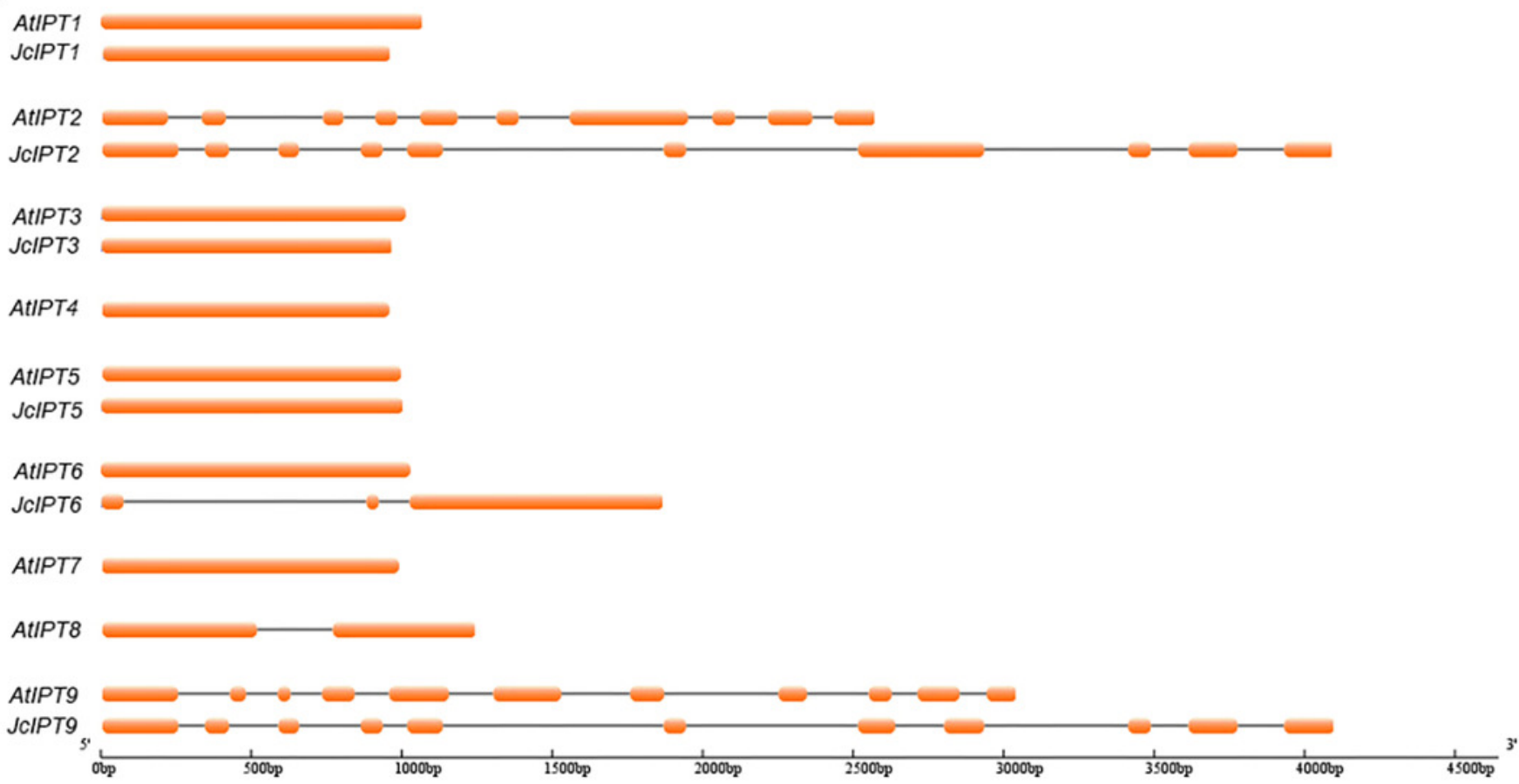

b

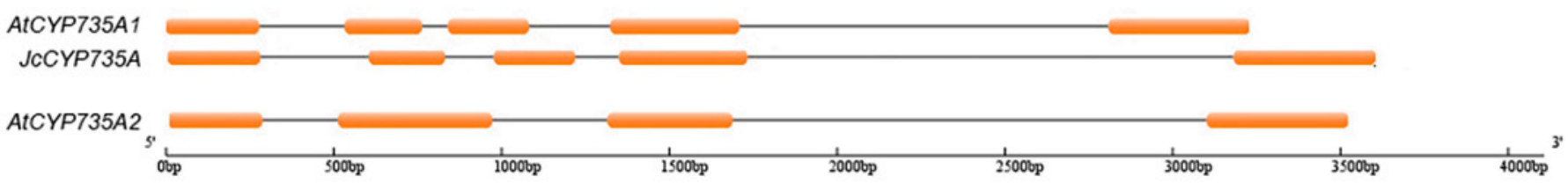

Legend: 


\section{Figure 3}

Genomic organization of $C K X$ family members in Jatropha and Arabidopsis.

At, Arabidopsis thaliana; Jc, Jatropha curcas.

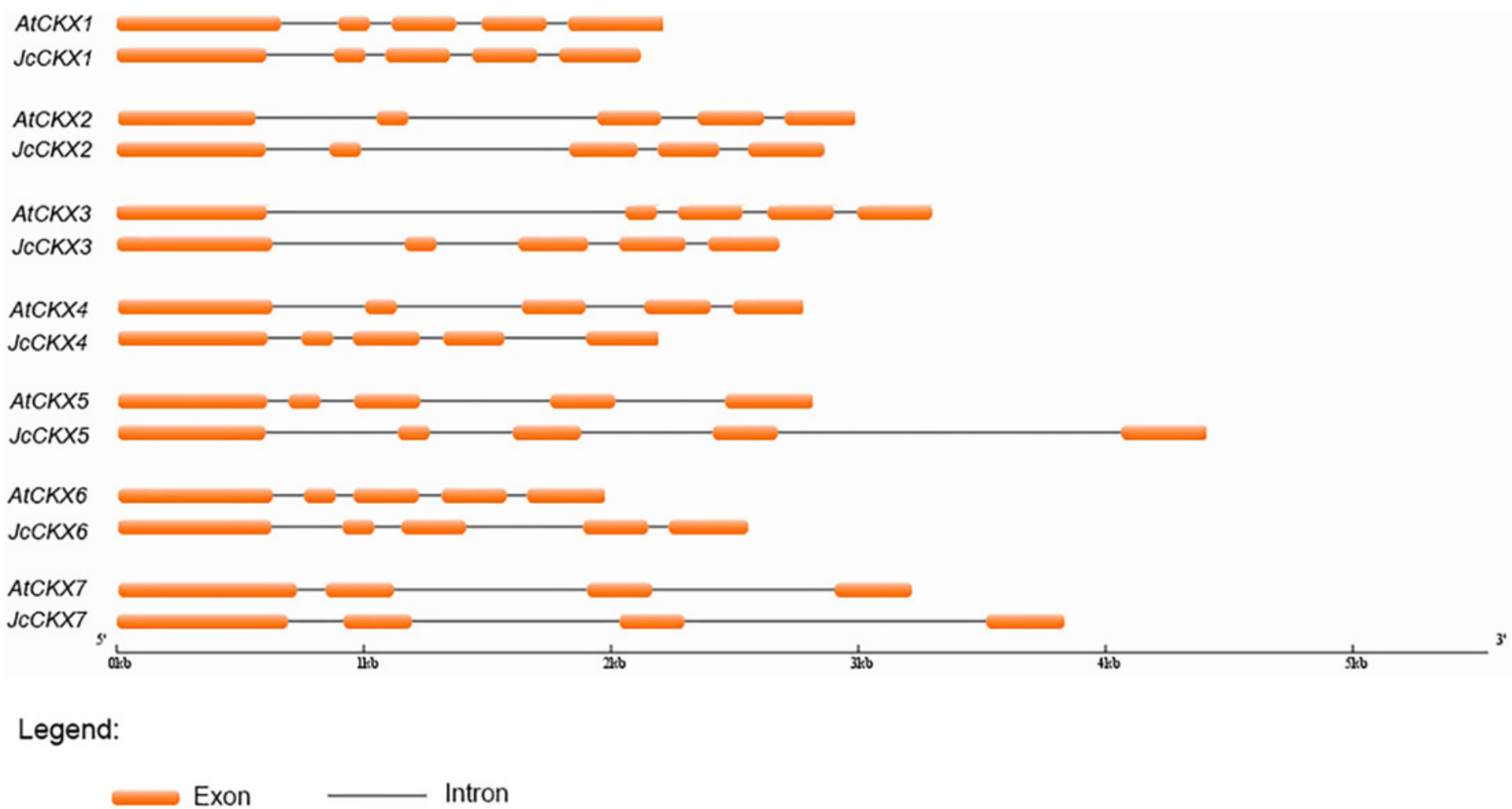




\section{Figure 4}

Neighbour-joining phylogenetic tree for IPT and CYP735A family members in various species.

(a) IPT family members; (b) CYP735A family members. At, Arabidopsis thaliana; Jc, Jatropha curcas; Os, Oryza sativa; Rc, Ricinus communis.
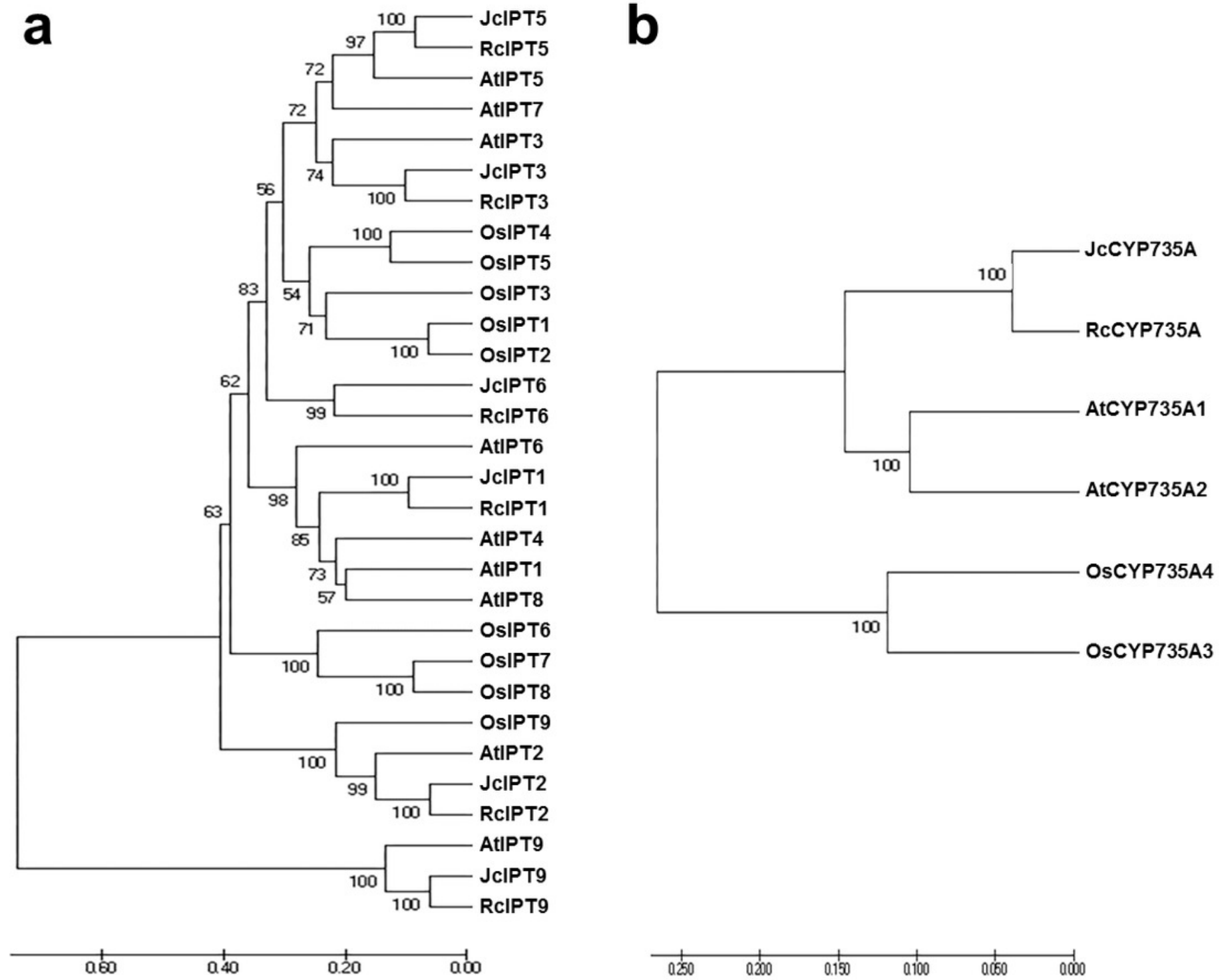
Figure 5

Neighbour-joining phylogenetic tree for CKX family members in various species.

At, Arabidopsis thaliana; Jc, Jatropha curcas; Os, Oryza sativa; Rc, Ricinus communis. 


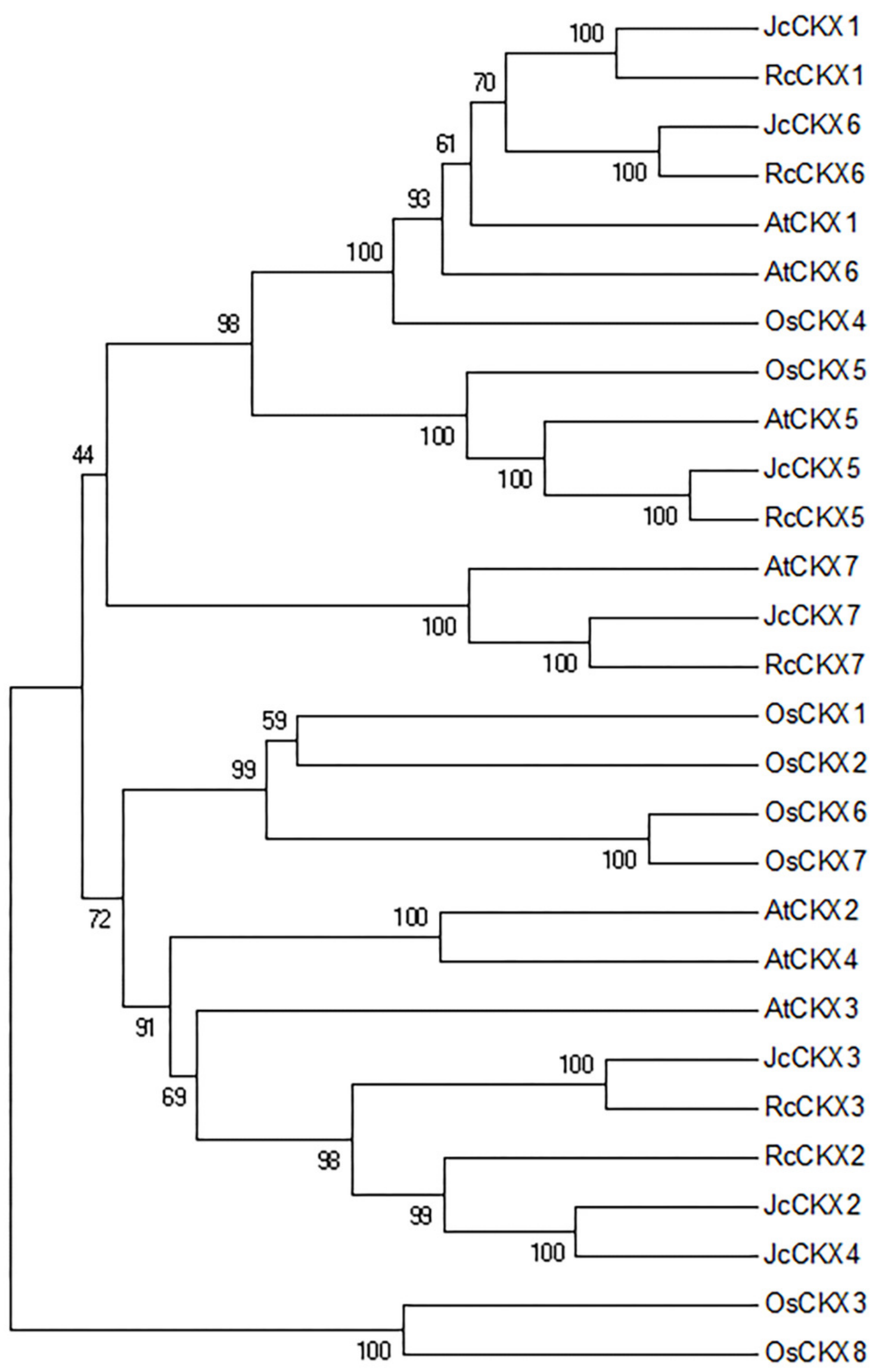




\section{Figure 6}

Expression of JCIPTs in various Jatropha tissues.

(a) to (f) are expression patterns of JcIPT1 , JcIPT2 , JcIPT3 , JcIPT5 , JcIPT6 , and JcIPT9, respectively. The qRT-PCR results were obtained from two independent biological replicates and three technical replicates for each sample. R, roots; SAM, shoot apical meristems; ST, stems; YL, young leaves; ML, mature leaves; FB, flower buds; FF, female flowers; MF, male flowers; FRU, fruits; SD, seeds; 0 DAP, unfertilized ovules; 10 DAP, seeds at 10 days after pollination; 20 DAP, seeds at 20 days after pollination; 30 DAP, seeds at 30 days after pollination; DAP, days after pollination.

a
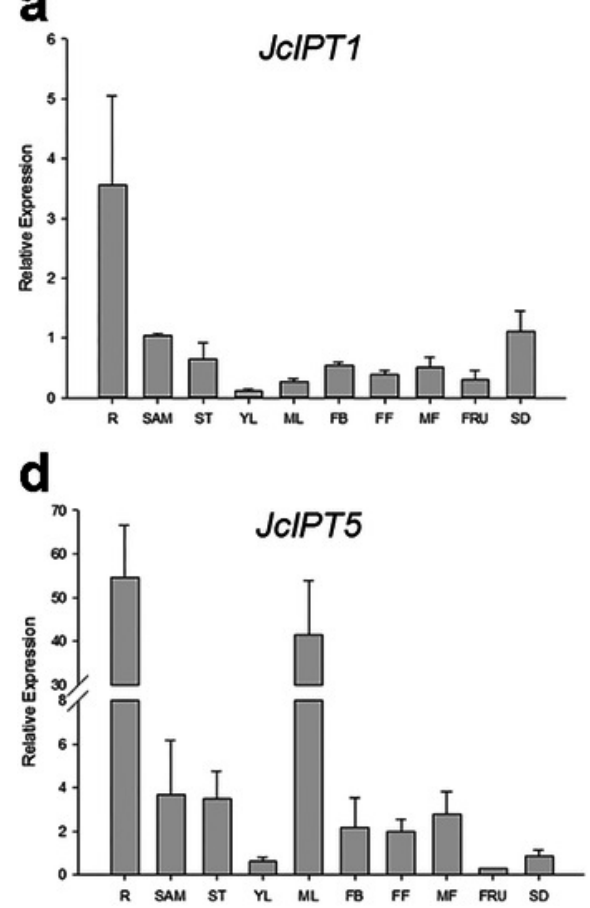

b

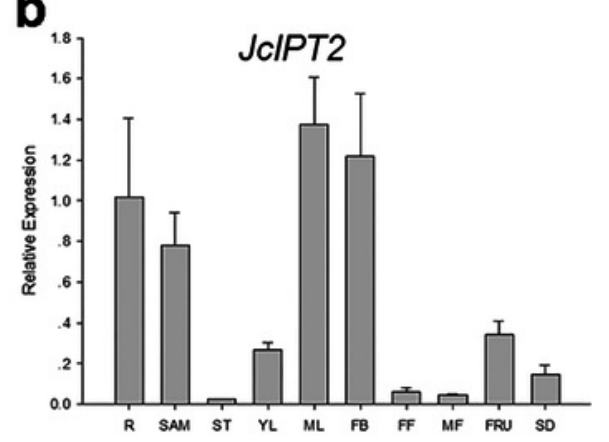

e

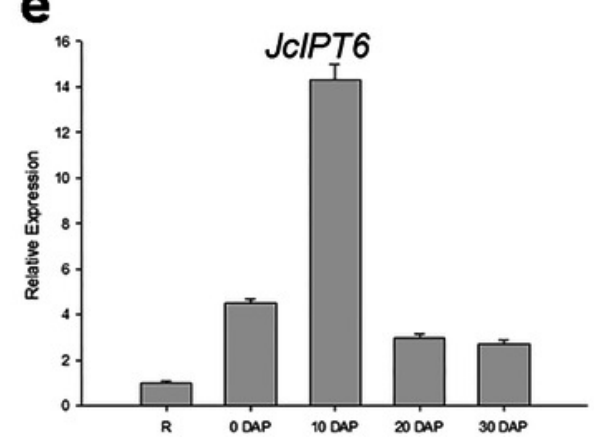

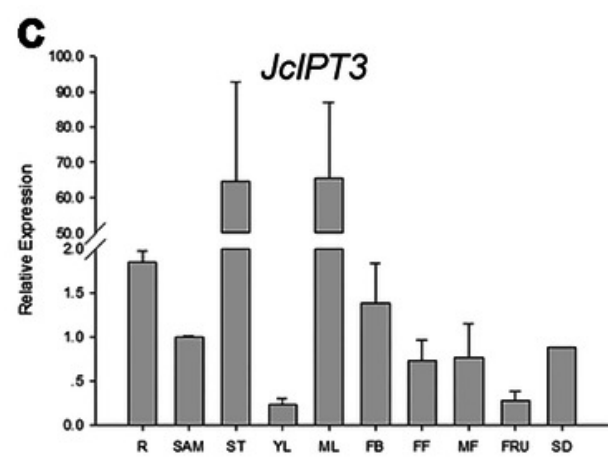

f

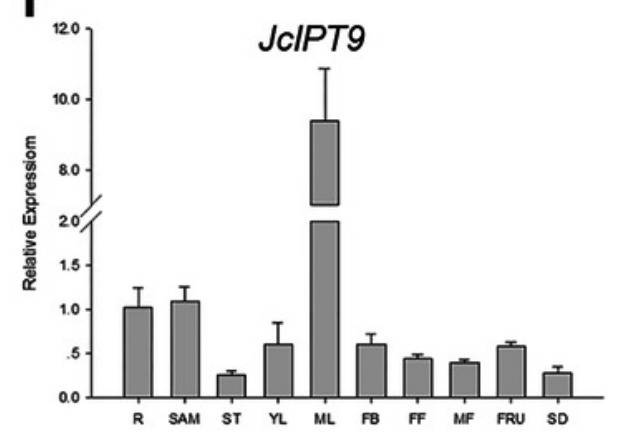




\section{Figure 7}

Expression of JCCYP735A and JcCKXs in various Jatropha tissues.

(a) to (h) are expression patterns of JcCYP735A , JcCKX1 , JcCKX2 , JcCKX3 , JcCKX4, JcCKX5, JcCKX6, and JcCKX7, respectively. Values in the Y-axis of (e) are displayed in scientific notation. e, exponent. The qRT-PCR results were obtained from two independent biological replicates and three technical replicates for each sample. R, roots; SAM, shoot apical meristems; ST, stems; YL, young leaves; ML, mature leaves; FB, flower buds; FF, female flowers; MF, male flowers; FRU, fruits; SD, seeds.
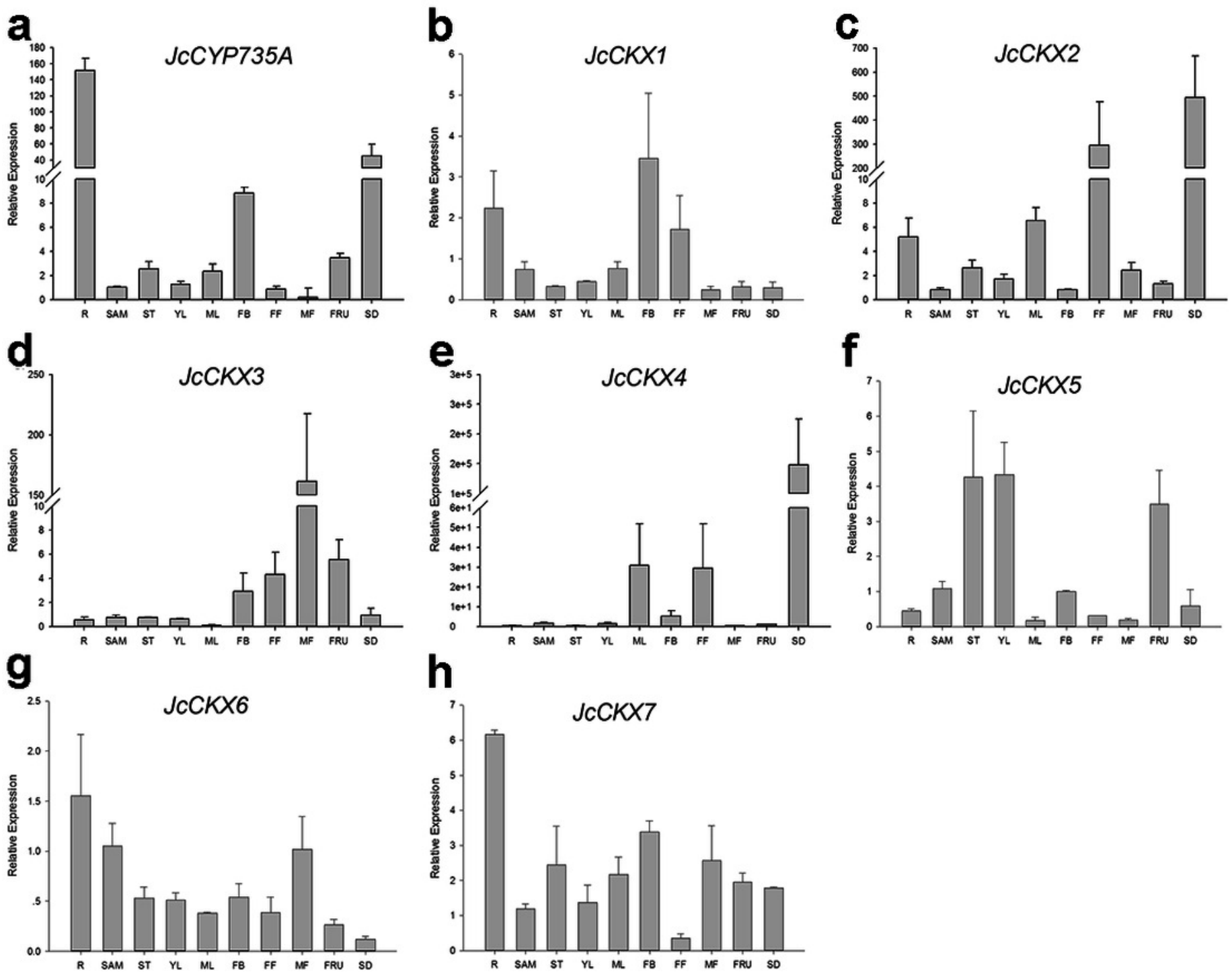


\section{Figure 8}

Generation and phenotypic variation of Jccyp735a mutants of Jatropha obtained by the CRISPR-Cas9 system.

(a) Different types of JCCYP735A mutation generated by the CRISPR-Cas9-mediated gene silencing in the progenies (T1 generation) of the three transgenic lines L1, L2, and L3. The blue characters show the selected target site sequences. The characters in purple box show the protospacer adjacent motif (PAM) sequences. The red character indicates the nucleotide insertion. WT, wild-type. (b-d) Cytokinin concentrations (b), the heights (c), and appearance (d) of the four-month-old seedlings (T1) of Jccyp735a mutant lines and WT. Error bars represent the standard deviation (SD) of three (b) or five (c) biological replicates. Asterisks indicate statistically significant differences compared with WT $(p<0.05)$. In $(d)$, bar $=5 \mathrm{~cm}$. 
a

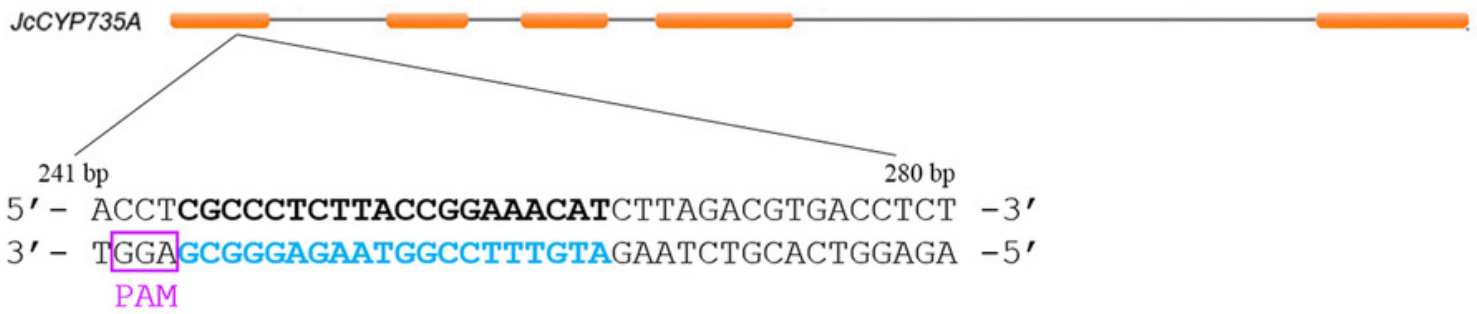

WT GCGTGGCCCTGAACCTCGCCC-TCTTACCGGAAACATCTTAGACGTGACCTCTTTTGTCTCCCA L1 GCGTGGCCCTGAACCTCGCC-----TACCGGAAACATCTTAGACGTGACCTCTTTTGTCTCCCA L2 GCGTGGCCCTGAACCTCGCCCСTCTTACCGGAAACATCTTAGACGTGACCTCTTTTGTCTCCCA L3 GCGTGGCC---------------------------------------TCTTTTGTCTCCCA Legend:

Exon Intron

b

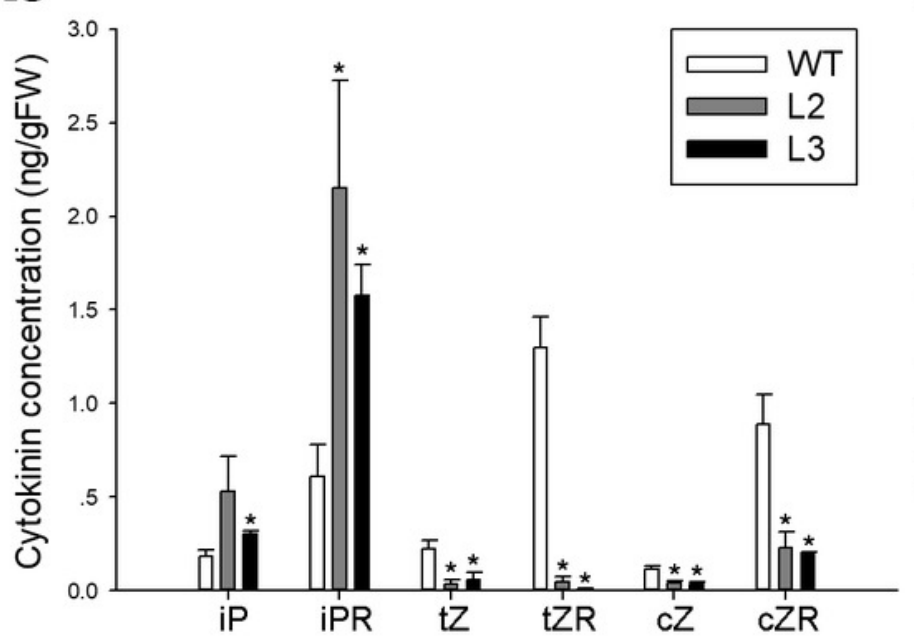

d
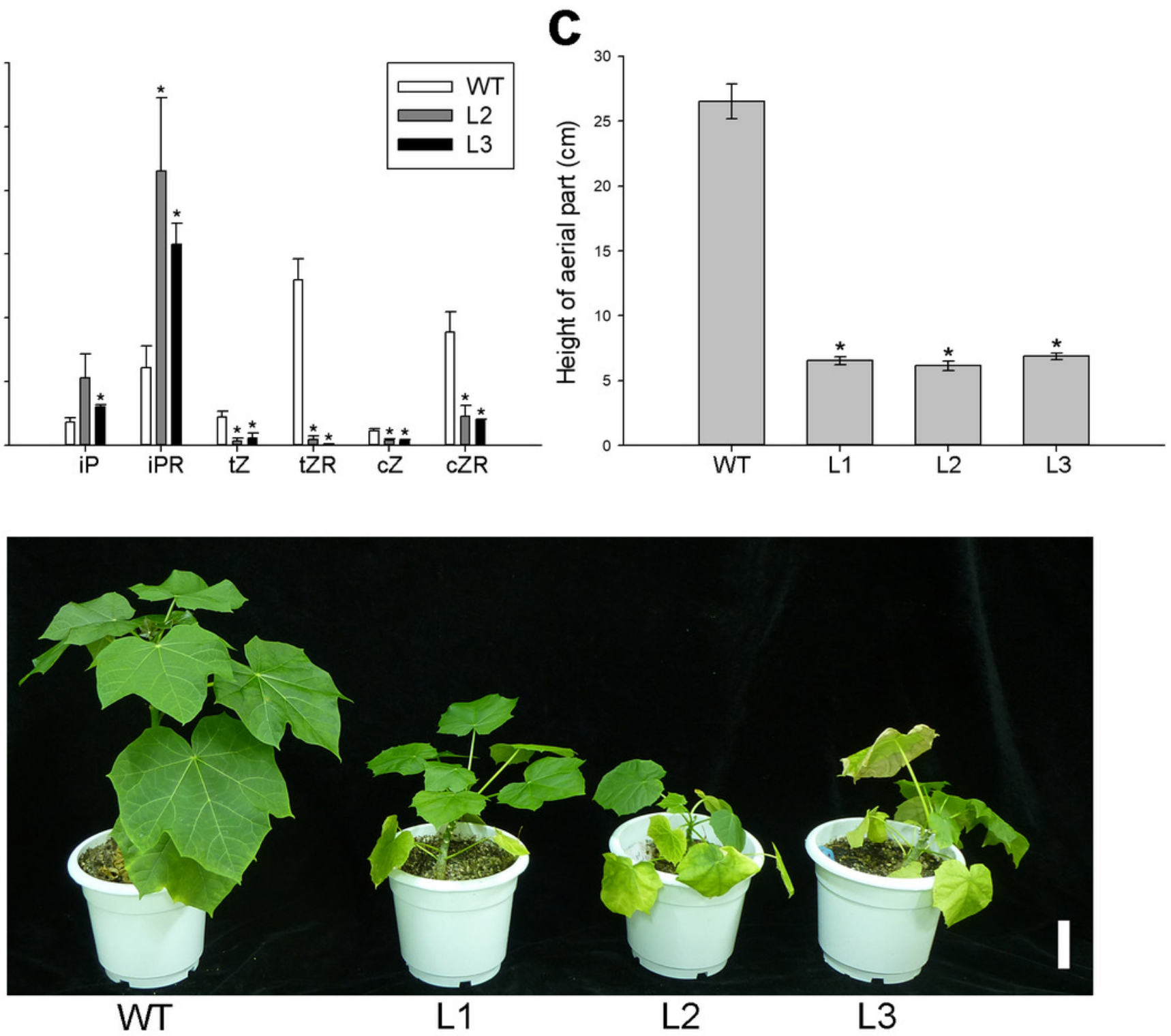


\section{Table $\mathbf{1}$ (on next page)}

Content of endogenous CKs in flower buds and seeds of Jatropha in different developmental stages (ng/gFW).

$\mathrm{FB1}$, flower buds of less than $5 \mathrm{~mm}$ in length; $\mathrm{FB} 2$, flower buds of 2-3 $\mathrm{cm}$ in length; 0 DAP, unfertilized ovule; $10 \mathrm{DAP}$, seeds of 10 days after pollination; $20 \mathrm{DAP}$, seeds of 20 days after pollination; DAP, days after pollination; N.D., not detected; N.Q., not quantified. 
1 Table 1. Content of endogenous CKs in flower buds and seeds of Jatropha in different developmental stages 2 (ng/gFW).

\begin{tabular}{ccccccccc}
\hline Samples & iP & iPR & iP9G & tZ & tZR & tZ9G & DZ & DZR \\
\hline FB1 & $\mathbf{0 . 1 4} \pm \mathbf{0 . 0 1}$ & $\mathbf{1 . 5 0} \pm \mathbf{0 . 0 3}$ & N.D. & $\mathbf{1 . 2 1} \pm \mathbf{0 . 0 3}$ & $\mathbf{1 2 . 5 5} \pm \mathbf{0 . 7 8}$ & N.Q. & $\mathbf{1 . 2 7} \pm \mathbf{0 . 0 9}$ & $\mathbf{9 . 2 4} \pm \mathbf{0 . 1 5}$ \\
FB2 & $\mathbf{2 4 . 8 6} \pm \mathbf{0 . 8 3}$ & $\mathbf{1 5 . 2 4} \pm \mathbf{0 . 2 4}$ & N.D. & $\mathbf{2 . 4 9} \pm \mathbf{0 . 1 7}$ & $\mathbf{0 . 8 9} \pm \mathbf{0 . 0 8}$ & N.D. & $\mathbf{3 . 3 1} \pm \mathbf{0 . 1 9}$ & $\mathbf{0 . 9 7} \pm \mathbf{0 . 0 3}$ \\
0 DAP & $\mathbf{3 . 9 7} \pm \mathbf{0 . 4 0}$ & $\mathbf{1 . 2 0} \pm \mathbf{0 . 0 7}$ & N.D. & $\mathbf{0 . 7 9} \pm \mathbf{0 . 0 8}$ & $\mathbf{0 . 0 8} \pm \mathbf{0 . 0 0 7}$ & N.D. & $\mathbf{0 . 5} \pm \mathbf{0 . 0 6}$ & N.D. \\
10 DAP & $\mathbf{3 . 9 6} \pm \mathbf{0 . 1 9}$ & $\mathbf{0 . 7 1} \pm \mathbf{0 . 0 2}$ & N.D. & $\mathbf{2 2 . 6 1} \pm \mathbf{2 . 4 2}$ & $\mathbf{1 . 5 6} \pm \mathbf{0 . 0 9}$ & $\mathbf{0 . 0 6} \pm \mathbf{0 . 0 0 1}$ & $\mathbf{6 . 4 2} \pm \mathbf{0 . 2 4}$ & $\mathbf{0 . 1 8} \pm \mathbf{0 . 0 2}$ \\
20 DAP & $\mathbf{0 . 3 1} \pm \mathbf{0 . 0 1}$ & $\mathbf{0 . 7 8} \pm \mathbf{0 . 0 5}$ & N.D. & $\mathbf{1 4 9 . 2 8} \pm \mathbf{1 1 . 6 0}$ & $\mathbf{3 9 . 7 6} \pm \mathbf{1 . 8 0}$ & $\mathbf{0 . 7 5} \pm \mathbf{0 . 0 3}$ & $\mathbf{2 8 . 9 0} \pm \mathbf{2 . 6 8}$ & $\mathbf{1 6 . 5 6} \pm \mathbf{0 . 8 6}$ \\
\hline
\end{tabular}

3 FB1, flower buds of less than $5 \mathrm{~mm}$ in length; FB2, flower buds of 2-3 $\mathrm{cm}$ in length; 0 DAP, unfertilized ovule; 410 DAP, seeds of 10 days after pollination; 20 DAP, seeds of 20 days after pollination; DAP, days after 5 pollination; N.D., not detected; N.Q., not quantified. 